Meta

Journal des traducteurs

Translators' Journal

\title{
La traduction des connaissances scientifiques en arabe : état des lieux, défis et perspectives
}

\section{Akram Odeh}

Volume 61, numéro hors-série, 2016

Sciences en traduction

Sciences in Translation

URI : https://id.erudit.org/iderudit/1038689ar

DOI : https://doi.org/10.7202/1038689ar

Aller au sommaire du numéro

Éditeur(s)

Les Presses de l’Université de Montréal

ISSN

0026-0452 (imprimé)

1492-1421 (numérique)

Découvrir la revue

Citer cet article

Odeh, A. (2016). La traduction des connaissances scientifiques en arabe : état des lieux, défis et perspectives. Meta, 61, 131-143.

https://doi.org/10.7202/1038689ar
Résumé de l'article

Comme ce fut le cas dans le passé, la traduction est, pour le monde arabe, la voie royale pour accéder aux connaissances modernes. Aussi l'état de la traduction de et vers l'arabe est-il d'actualité depuis deux décennies. Cette contribution confronte diachroniquement les différents états des lieux de ce transfert. Elle cherche à mettre au jour les obstacles qui entravent son épanouissement tout en plaçant sa problématique dans un contexte socioéconomique et politique. L'héritage culturel arabe de jadis étant inadéquat pour le $\mathrm{XXI}^{\mathrm{e}}$ siècle, cette étude souligne l'importance /de s'inspirer des expériences réussies des autres nations modernes pour traduire les savoirs actuels. 


\title{
La traduction des connaissances scientifiques en arabe: état des lieux, défis et perspectives
}

\author{
AKRAM ODEH \\ Université de Jordanie, Jordanie \\ akramodeh10@gmail.com
}

\begin{abstract}
RÉSUMÉ
Comme ce fut le cas dans le passé, la traduction est, pour le monde arabe, la voie royale pour accéder aux connaissances modernes. Aussi l'état de la traduction de et vers l'arabe est-il d'actualité depuis deux décennies. Cette contribution confronte diachroniquement les différents états des lieux de ce transfert. Elle cherche à mettre au jour les obstacles qui entravent son épanouissement tout en plaçant sa problématique dans un contexte socioéconomique et politique. L'héritage culturel arabe de jadis étant inadéquat pour le $\mathrm{XX|}$ siècle, cette étude souligne l'importance /de s'inspirer des expériences réussies des autres nations modernes pour traduire les savoirs actuels.
\end{abstract}

\begin{abstract}
As was the case in the past, translation is for the Arab World the high road to modern knowledge. Thus, the status of translation from and into Arabic has been a topic of discussion for two decades. This paper compares diachronically the different status of this transfer. It seeks to bring to light barriers to its development while putting its problems in a socioeconomic context inherent to the Arab world. Arabic cultural heritage of the past is inadequate for the $27^{\text {st }}$ century. This study highlights the importance to learn from successful experiences of other modern nations to reflect current science.
\end{abstract}

\section{MOTS-CLÉS/KEYWORDS}

traduction dans le monde arabe, transfert des connaissances, état des lieux translation in the Arab world, knowledge transfer, state of things

\section{Introduction}

La traduction demeure le principal moyen d'importer toutes sortes de connaissances scientifiques dans la langue arabe. Aussi l'état de la traduction de et vers l'arabe estil d'actualité depuis deux décennies. Certains rapports d'organismes internationaux et panarabes suggéraient, comme l'a résumé Jacquemond, qu'en arabe «on traduit toujours trop peu, trop tard, jamais les livres qu'il faudrait traduire, et jamais comme il le faudrait» (2012: s.p.). D’autres dressent un état des lieux plutôt positif, mentionnant qu' "au cours des dix dernières années de 1500 à 2000 titres par an » sont traduits en arabe (Glasson Deschaumes 2012: 15).

Néanmoins, il serait judicieux de rappeler que le transfert des savoirs dans la communauté linguistique arabo-musulmane ne concerne pas un seul état homogène. Au contraire, il s'agit ici d'un monde complexe composé de toutes sortes de régimes politiques et économiques, ainsi que de communautés linguistiques, culturelles et religieuses (Béligh 2013: 17). Certes, le monde arabe est un ensemble géographique très étendu, mais cet espace est parsemé de contradictions en termes de démographie, d'éducation, d'analphabétisme et de richesse des ressources naturelles. 


\section{Une expérience déjà vécue}

Le monde arabe serait-il apte à relever le défi de traduire les connaissances scientifiques modernes? Ce dernier n'en est pas à sa première expérience de transfert des savoirs vers la langue et la culture arabes. Aux VIII et $\mathrm{IX}^{\mathrm{e}}$ siècles, les Arabes ont entamé un projet de traduction d'ouvrages scientifiques et philosophiques perses, indiens et grecs, qui ont été intégrés à la civilisation arabe pour aboutir à ce qu'on appelle traditionnellement «le mouvement d'édification scientifique (Salama-Carr 1990 : 30). La Maison de sagesse abbasside est considérée comme l'une des premières écoles de traduction de l'histoire humaine (Yasa 2003: 213). Ce mouvement a permis la constitution d'une brillante civilisation pendant le Moyen-Âge, à la fin duquel le centre de traduction mondial s'est déplacé de Bagdad à Tolède (Delisle 1995: 123). Quelques siècles plus tard, «le mouvement de résurrection scientifique» voit le jour au XIX ${ }^{\mathrm{e}}$ siècle. Ce mouvement s'est édifié en ayant recours à la traduction des œuvres scientifiques européennes, rédigées notamment en français et en anglais. Mais, cette entreprise n'a pas eu les retombées escomptées. Le monde arabe vivait un immobilisme scientifique dû au déclin de l'Empire ottoman et allait entrer dans l'époque de la colonisation et de la division géopolitique.

De nos jours, les efforts de traduction en arabe des savoirs scientifiques relèvent selon Cheddadi (2009: 98) de quatre types d'entreprises. D'abord, il y a les traductions individuelles et institutionnelles au niveau local d'un pays. Il s'agit d'initiatives d'individus, d'universitaires ou de maisons d'édition locales. Viennent ensuite les transferts initiés et pilotés par les gouvernements à l'échelle nationale. Il est question ici des centres spécialisés en traduction, issus de projets de traduction financés par l'État. Il existe aussi les productions d'organismes panarabes de la Ligue arabe, comme l'Organisation arabe pour l'éducation, la culture et les sciences (ALECSO). Les efforts de ces organismes portent sur la traduction, l'uniformisation de la terminologie scientifique arabe et l'édition d'ouvrages terminographiques. Enfin, il y a les traductions initiées et financées par des institutions internationales et les ambassades étrangères basées dans les capitales arabes. Le Goethe Institut a subventionné entre 1995 et 2011 la traduction de l'allemand vers l'arabe de 89 titres (Naggar 2011 : s.p.). Il faudrait également mentionner les traductions soutenues par l'Union soviétique, qui ont enrichi la bibliothèque arabe de nombreux ouvrages, notamment ceux qui répandent l'idéologie communiste. Le dernier projet de traduction serait le programme Tradarabe (MAE 2013: s.p.), projet de soutien à la traduction du français vers l'arabe qui comprend une base de données recensant les titres traduits du français vers l'arabe, leurs éditeurs et leurs traducteurs. Il a été initié et piloté par le Quai d'Orsay.

\section{L'état actuel de la traduction dans le monde arabe}

La dernière décennie a connu des débats d'une ampleur sans précédent sur l'état actuel de la traduction de et vers l'arabe et sur la circulation des connaissances. Il est possible de classer les études faites au cours de cette période en deux catégories. D’une part, nous trouvons les études alarmistes et pessimistes. C'est le cas entre autres des rapports du Programme des Nations Unies pour le Développement (PNUD) de 1999 et de 2003 et du rapport de l'UNESCO de 2006. D'autre part, notons les recherches 
rassurantes qui font un état des lieux plutôt positif, tout en soulignant les handicaps du transfert des savoirs en langue arabe. C'est le cas par exemple de l'étude de la Fondation du Roi Abdul-Aziz réalisée en 2012, dont Richard Jacquemond fait le rapport dans Transeuropéennes (Jacquemond 2012).

\subsection{Les rapports alarmistes}

Le PNUD a publié deux rapports, en 1999 et en 2003, très négatifs sur la traduction de et vers l'arabe, lesquels ont été très contestés par l'élite arabe et arabisante. Celui de 1999 affirme que "le nombre total d'ouvrages traduits dans le monde arabe depuis le règne du calife Al-Ma'mûn [786-833 apr. J.-C.] au XI siècle est d'environ 10000 , soit l'équivalent du volume de traductions réalisées en Espagne en un an (Badran 2006: 171). Le rapport de 2003 affirme que «la Grèce, pays de onze millions d'habitants vingt-cinq fois moins peuplé que l'ensemble du monde arabe (285 millions d'habitants en 2003), traduirait dans sa langue plus de livres que les vingt-deux États arabes» (PNUD 2003: s.p.).

D’autres études encore plus catastrophistes sont citées par Janjar (2013). La première, tirée de la recherche parue en 1999 de M. Galal Shawqi, souligne que "pour l'année 1986, par exemple, il s'agit de 268 titres traduits dans le monde arabe contre 462 titres en Israël, dont la population est cinquante fois moins importante que celle du monde arabe» (Shawqi 1999: 2). La seconde est tirée d'un ouvrage intitulé Plan arabe pour la traduction, publié par l'Organisation arabe pour l'éducation, la culture et les sciences (ALECSO 1997: 124), organisme faisant partie de la Ligue arabe. Cette étude souligne que la quantité d'ouvrages traduits en arabe depuis le califat abbasside serait égale au nombre de livres traduits en une seule année en Espagne (ALECSO 1997: 124).

En 2006, l'UNESCO a publié un rapport sur la science dans le monde arabe. Son auteur, Badran, dresse un portrait pessimiste concernant les réalités des sciences dans la sphère arabophone. Il met l'accent sur l'importance de la traduction pour remédier à son sous-développement. Quand Badran examine l'état de la traduction dans l'arabophonie, il avance des statistiques et évoque des comparaisons inquiétantes:

Aujourd'hui, le nombre d'ouvrages traduits en arabe est d'environ 5 pour 1 million d'habitants. À titre de comparaison, en Espagne, 920 ouvrages par million d'habitants sont actuellement traduits en espagnol. Pour prendre un autre exemple, les auteurs arabes publient environ 6500 ouvrages par an dans la région arabe, contre 102000 en Amérique du Nord. (Badran 2006: 171-172)

Certains chercheurs ont réfuté les études précitées, et les arguments sont nombreux. Jacquemond (2007) cite le projet national égyptien de traduction à l'époque de Nasser, qui a abouti à la traduction de 1000 livres entre 1955 et 1970. Le projet de la Fondation M. Ben Rachid Al-Maktoum, l'émir de Dubaï, qui visait la traduction de 1000 ouvrages en trois ans, soit un livre par jour, et le projet Kalimah lancé en 2007 par les autorités culturelles de l'Émirat d'Abu Dhabi, qui a traduit 500 livres jusqu'en 2010, constituent, selon Janjar (2013) de bons témoins du mouvement de traduction. On peut également à cela ajouter d'autres programmes comme les travaux de l'Organisation arabe de la traduction au Liban, le Projet national de traduction au Caire, le Centre national de la traduction en Tunisie, la Fondation Shomane en Jordanie. 
Jacquemond (2007: 178) explique le ton pessimiste dominant concernant l'état de la traduction de et vers l'arabe par le fait que les sources des statistiques catastrophistes sont des organismes internationaux comme le PNUD ou encore l'Index Translationum de l'UNESCO, qui est un répertoire et une bibliographie de livres traduits dans le monde entier. Or, les données incluses dans les bases de données de ces institutions sont fournies par les autorités officielles des États arabes, dont la collecte statistique particulièrement parcellaire et aléatoire est symptomatique d'un secteur peu professionnalisé. Il souligne en outre que ces organismes ont commis la grave erreur de comparer la situation de transfert traductionnel en arabe avec celle des pays européens et non avec celle des pays émergents d'Asie et d'Amérique latine, deux aires culturelles auxquelles le monde arabe est constamment confronté pour déterminer les autres indicateurs de développement, notamment le développement humain.

Par exemple, en croisant le total des traductions publiées (Index, période 1978-2003) et la population actuelle, on obtient certes 28 traductions par million d'habitants pour le Monde arabe («moins d'un livre par an et par million d'habitants», comme dit le rapport), mais aussi 17 pour l'Indonésie, 19 pour la Thaïlande, 50 pour la Malaisie, ou encore 424 pour la Corée et 702 pour le Japon, mais seulement 132 pour les ÉtatsUnis, 2250 pour la France, 2750 pour l'Allemagne et près de 10000 pour le Danemark! Bref, les choses sont plus complexes que ne le donnent à croire le rapport du PNUD et les statistiques de l'UNESCO. (Jacquemond 2007: 180)

À leur tour, Dessa et Janjar (2010: 2) critiquent non seulement ces statistiques, mais aussi les controverses sur les statistiques du nombre de livres traduits de et vers l'arabe. Pour ces auteurs, la question de la traduction est un enjeu culturel majeur dont les obstacles sont variés et de diverse nature. Néanmoins, ils soulignent que, malgré le caractère erroné et contestable des statistiques des instances internationales, c'est grâce à celles-ci qu'une prise de conscience de l'importance et du retard pris en matière de transfert des connaissances scientifiques a été généralisée, suscitant de vifs débats au moins auprès des élites. Au-delà de la question de la traduction, Dessa et Janjar, tout comme Jacquemond, rappellent que les chiffres avancés du nombre de livres traduits montrent bien la faillite des systèmes statistiques arabes.

\subsection{Les rapports plus rassurants}

L'une des premières institutions panarabes ayant travaillé exclusivement sur la question de la traduction dans l'arabophonie est la Fondation du Roi Abdul-Aziz, à Rabat. Cette institution a mené de nombreuses recherches en arabe portant sur l'état de la traduction de et vers l'arabe, dont les conclusions ont été publiées en français par H. Dessa et M. S. Janjar dans le rapport État des lieux de la traduction en Méditerranée édité par la Fondation Anna Lindh et par Transeuropéennes.

Dessa et Janjar (2010:3) rendent compte de l'état actuel de la traduction en arabe des œuvres de sciences humaines et sociales en se basant sur les études et les corpus les plus récents réalisés par ladite Fondation. L'importance de leur étude tient d'une part au fait qu'elle porte sur le transfert des savoirs des sciences humaines et sociales, véritable pilier de tout développement culturel et, d'autre part, au fait que leur rapport puise ses «données à partir d'un catalogage des ouvrages publiés et non pas uniquement à partir de bulletins des agences bibliographiques nationales ou des catalogues 
des éditeurs» (Dessa et Janjar 2010: 7). Les résultats de ces enquêtes montrent une évolution indéniable.

La consultation de la base de données de la Fondation au cours des mois de mai-juin 2010, permet de recenser quelque 2670 titres publiés par des éditeurs arabes durant la décennie 2000-2009, soit une moyenne annuelle de 268 titres. Pour la décennie précédente, la moyenne était de 198 titres, soit une augmentation de 34\%. [...] [L]a traduction des ouvrages de sciences humaines et sociales représenterait entre $13 \%$ et $18 \%$ de l'ensemble des traductions arabes. (Dessa et Janjar 2010: 6)

Cette recherche met l'accent sur certains problèmes dans le transfert en arabe des sciences humaines et sociales, comme le non-respect des droits d'auteur ou le peu d'intérêt vis-à-vis du transfert d'œuvres espagnoles, allemandes, russes et asiatiques. Les deux auteurs soulignent plusieurs phénomènes qui méritent d'être signalés: l'importance relative de la traduction d'ouvrages de sciences sociales et humaines du français en arabe, car $33 \%$ des titres traduits l'ont été du français contre $48 \%$ de l'anglais; l'épanouissement du transfert des ouvrages iraniens en arabe; la domination de la traduction des deux auteurs incontournables que sont Noam Chomsky et Edward Saïd; et une tendance à la professionnalisation de la traduction en sciences sociales. Les traces de cette professionnalisation se manifestent d'une part par l'inclusion dans les textes traduits d'un "appareil critique " comprenant une révision et une introduction fournissant au lecteur des informations sur le livre traduit et son auteur et, d'autre part, par la traduction de textes intermédiaires résumant les grandes œuvres classiques et fondamentales.

Dans une perspective d'interculturalité, Transeuropéennes et la Fondation Anna Lindh, en partenariat avec une quinzaine d'institutions appartenant à des pays euroméditerranéens, ont lancé et piloté un projet collectif de deux ans, de 2010 à 2012, visant à faire l'état des lieux de la traduction en Méditerranée. Le mouvement de la traduction entre l'arabe, le turc, l'hébreu et les langues de l'Union européenne y occupe une place importante. Il s'agit d'un gigantesque projet sans précédent: 69 études par paire de langues et par thème réalisées par 64 traducteurs et chercheurs. Palliant ainsi la carence en études critiques des échanges culturels euro-méditerranéens, ce projet "prend en compte toute la chaîne de traduction: auteurs, traducteurs, éditeurs, libraires, bibliothèques, programmes d'aide à la traduction, médias " (Glasson Deschaumes 2012: 9). Il va sans dire qu'il a abouti à une meilleure connaissance de l'état actuel de la traduction dans cette zone, ce qui permettra de définir des stratégies et d'envisager des programmes dans l'avenir.

Les résultats des études menées par Transeuropéennes sur l'état actuel de la traduction de et vers l'arabe sont positifs: «les estimations se rejoignent sur une moyenne de livres traduits au cours des dix dernières années de 1500 à 2000 titres par an, la moyenne étant plus faible sur la décennie antérieure» (Glasson Deschaumes 2012: 15). Les deux principales langues sources sont l'anglais et le français. Les thèmes ayant été les plus traduits relèvent des sciences sociales. Les pays arabes qui traduisent le plus sont le Liban, la Syrie, l'Égypte et le Maroc. Cette évolution est attribuée, toujours selon l'étude en question, à différents facteurs, comme le développement de l'édition privée et l'émergence d'organismes arabes de traduction professionnelle. 


\section{Défis et difficultés}

Les études susmentionnées, les pessimistes tout comme les optimistes, dressent le bilan d'un certain nombre de difficultés entravant la constitution d'une masse critique de traductions apte à amorcer un début de renaissance scientifique arabe. Ces difficultés sont nombreuses et de nature diverse. Le traitement de l'ensemble de ces obstacles dépasse le cadre de cette contribution. Néanmoins, il est possible d'étudier quelques aspects de ces défis sous deux angles. D'abord, il faut souligner les problèmes inhérents à toute la chaîne de l'industrie de la traduction: métier du traducteur, maisons d'édition et gestion de la traduction. Ensuite, le contexte socioculturel dans la sphère arabe n'est pas propice à la traduction, faute tout d'abord de stabilité politique, de paix sociale, d'une politique d'arabisation rationnelle et progressive de l'enseignement scientifique universitaire, et faute aussi de l'arabisation de beaucoup de logiciels et d'outils informatiques.

Toutefois, chercher à lever les difficultés mentionnées pour réussir le transfert des connaissances scientifiques dans le monde arabe devrait se faire en s'inspirant de modèles. Le premier modèle qui pourrait être suivi serait celui de l'apogée de la civilisation arabo-musulmane. Ce dernier ne semblerait pas être fructueux (Zakariya 1990; Baccouche 2000). Certes, les contextes et les conditions ayant permis l'épanouissement de la civilisation arabe de jadis n'ont pas de traces dans le monde arabe actuel. À titre d'exemple, le symbole de l'apogée de la traduction fut le calife Al-Ma'mûn, qui encourageait les traducteurs et les payait cher. Cet homme régnait seul sur un empire stable et harmonieux. Les différents groupes linguisticoreligieux jouissaient d'une paix sociale qui permettait à chacun de s'épanouir et à la communauté d'avancer à tous les niveaux. Or, le monde arabe actuel se trouve à l'opposé. Il est instable, paradoxal, déchiré et meurtri par des conflits internes et externes de toutes sortes: économiques, politiques, religieux, linguistiques. Si un traducteur abbasside pouvait commencer à traduire un manuscrit à Constantine, en Algérie, et le finir à Fès, au Maroc, de nos jours, les frontières entre ces deux États sont fermées depuis bientôt trois décennies. Ni traducteur, ni traduction n'y circulent.

Baccouche explique que ni l'exemple du patrimoine arabe, ni celui de la Renaissance européenne ne constituent de bons exemples pour la traduction des savoirs modernes en arabe:

Il est évident que, dans les deux premiers cas [la traduction arabe du grec et la traduction européenne de l'arabe], il s'agissait de traduire le produit intégral d'une civilisation qui a cessé de se développer par la création continue. Il était donc aisé d’en faire le tour, d'assimiler ce produit et de s'engager dans l'action de dépassement et de création. Dans le dernier cas [la traduction des savoirs actuels en arabe], la traduction s'attaque à un produit en extension, qui progresse et se développe à un rythme beaucoup plus rapide que celui de sa traduction. D’autre part, cette action modernisante entamée au début du XIX ${ }^{e}$ siècle, notamment en Égypte, en Syrie, au Liban et en Tunisie, a vu son élan brisé par la colonisation qui a remplacé l'arabe par le français et l'anglais, devenus langues véhiculaires, de culture et d'enseignement. (Baccouche 2000: 398)

Par conséquent, il faudrait s'inspirer des expériences vécues et des modèles réussis de l'histoire récente, celle qui correspond à la mémoire des arabophones vivants. Les modèles des nouveaux pays émergents qui peuvent être géographiquement et culturellement lointains de l'arabophonie, mais dont l'expérience est plus proche du monde arabe et tangible pour lui, constituent un point de comparaison 
plus réaliste. Les Arabes et les Européens ont mis des siècles pour réussir ce que les Japonais ont réalisé en un seul; les pays émergents comme la Corée et la Turquie sont en passe d'accomplir en peu de décennies ce que les Japonais ont parachevé en un siècle.

\subsection{Les défis traductologiques}

Au-delà de son rôle primordial dans le transfert des connaissances dans le monde arabe, la traduction prend de nos jours une importance sans précédent comme médiatrice dans l'intercompréhension et la communication interculturelle entre l'espace arabe et le reste du monde, notamment l'Occident. Certes, le début de ce millénaire a été marqué par le déclenchement de la guerre contre le terrorisme suite aux tragiques évènements du 11/9, revendiqués par Al-Qaïda.

$\mathrm{Au}$ lendemain de ce grave incident, un climat de déficit de confiance, de suspicion et de rejet presque généralisé de tout ce qui pouvait se rapporter à l'islam et aux musulmans avait créé auprès d’une majorité de ces derniers une réaction de repli considérable (Basalamah 2011: 115-116)

L'avènement du printemps arabe a encore contribué à l'élargissement du fossé de l'incompréhension dans la communication interculturelle entre l'Orient arabe et l'Occident. En s'inspirant des valeurs démocratiques occidentales, les révolutions arabes ont à leurs débuts renversé des dictatures, mais elles ont aussi abouti à des guerres civiles et donné naissance à de nouvelles organisations islamistes très fanatiques, qui sèment la mort dans une grande partie du monde arabe.

Par conséquent, un grand effort de traduction doit être fait dans l'urgence «sur le rapport à l'islam, aux musulmans ainsi que sur l'islam lui-même» (Basalamah 2011: 115) pour répondre aux légitimes questionnements, doutes et inquiétudes soulevés par les non musulmans au sujet de cette foi.

L'entreprise de traduire en langues étrangères les productions culturelles et religieuses pouvant refléter la véritable image de l'Islam et de ses fidèles et permettre de les distinguer des interprétations extrémistes et des comportements sanglants de certains musulmans est une tâche épineuse et de longue haleine. Néanmoins, la traduction en tant quacte de communication, médiatrice entre les langues et les cultures, demeure une voie incontournable à emprunter pour jeter des ponts de compréhension mutuelle entre le monde arabe et le reste du monde, pourvu qu'elle ne soit pas muselée par la censure. Certes, traduire est un double acte de communication qui consiste à transférer au lecteur cible le contenu conceptuel et culturel du message source pour rapprocher le soi de l'autre. Maîtriser seulement les langues de travail ne permet pas au traducteur de remplir pleinement sa fonction de médiateur. Il est censé aussi connaître les références et les représentations culturelles vécues et partagées dans la réalité sociale, idéologique, politique de la culture source pour pouvoir les transposer et les expliquer à son lectorat cible. En effet, il est supposé non seulement interpréter le dit explicite et manifeste dans un énoncé, c'est-à-dire le sens véhiculé par les mots, mais aussi le non-dit culturel implicite du message, c'est-à-dire tout ce que le sens des mots ne dit pas comme le présupposé, le sous-entendu, l'allusion et la connotation qui doivent intervenir dans le calcul du sens.

Le rapport de Transeuropéennes de 2012 dresse une longue liste de défis concernant la traduction et les différents acteurs impliqués dans cette activité, entre autres 
la professionnalisation tardive du traducteur, qui n'a pas encore le statut, la reconnaissance et la rémunération qui lui reviennent de plein droit. Par exemple, dans divers pays arabes comme la Jordanie, il n'existe pas de réglementation spécifique concernant le métier de traducteur assermenté et son processus d'homologation en matière de certification, de formation, d'expérience, de déontologie, entre autres.

Cette profession manque en outre de reconnaissance sociale. La profession de traducteur n'est pas en général perçue comme un véritable métier; c'est un «petit emploi» complémentaire pour arrondir ses fins de mois. La faible rémunération du traducteur, notamment par les maisons d'édition, entrave souvent l'édition d'ouvrages traduits de qualité. Une page peut se négocier à dix dollars en Syrie ou en Égypte et à trente dans le Golfe.

Beaucoup de traductions sont réalisées, selon Transeuropéennes, sans respecter les droits d'auteur. Il n'est pas rare qu'une œuvre traduite soit commercialisée sans révision ou préface, au point de ne pas afficher le titre d'origine ou la langue source de la version arabe. Jacquemond (2012: s.p.) note que la majorité des maisons d'édition de l'arabophonie sont de petite taille, peu professionnelles et étatiques. Cela signifie des moyens financiers limités, le non-respect des droits d'auteurs, la qualité d'édition médiocre et le manque de compétence dans la communication et la commercialisation du livre traduit.

Depuis leur indépendance, les pays arabes n'ont pas réussi à mettre en place une stratégie de traduction ni au niveau panarabe, ni au niveau des régions, ni au niveau national. À cela s'ajoute un manque de coordination et de communication entre les maillons de la chaîne professionnelle de la traduction. Comme on n'est pas au courant de ce qui se fait en matière de traduction, il arrive qu'on traduise un livre déjà traduit. Certes, l'une des caractéristiques du monde arabe est que ses liens avec l'étranger sont plus solides et constants qu'entre les entités qui le composent. Cela est valable non seulement en traduction, mais aussi en ce qui a trait aux échanges commerciaux, politiques et culturels.

Enfin, la question de la traduction tardive des œuvres vers l'arabe est un véritable problème, notamment quand il s'agit de transférer des ouvrages scientifiques fondateurs. Ces derniers deviennent obsolètes peu d'années après leur parution; la traduction tardive entrave et complique le suivi de l'évolution des idées qui en découlent. La traduction de l'œuvre fondatrice de la linguistique moderne, le Cours de linguistique générale de Saussure, en est un exemple éloquent. Cet ouvrage a été publié en 1917. Néanmoins, sa traduction vers l'arabe n'est entreprise qu'au début des années 1980 : en moins de dix ans, l'arabophonie a connu cinq traductions successives, parfois à une année de différence. Les versions tunisienne, marocaine et syrienne ont été traduites du français; les traductions palestinienne et irakienne, à partir la version anglaise de Baskin. Dans deux versions, la qualité de la traduction était déplorable. Sur le plan des connaissances, le lecteur arabe a été, durant six décennies, privé des bases fondatrices du structuralisme. Au plan terminologique, les conséquences étaient néfastes pour le transfert de la terminologie linguistique moderne en arabe (Odeh 1998: 165).

L’œuvre à traduire est volumineuse, variée et évolutive. Janjar (2013: 4) souligne que les résultats escomptés porteront leurs fruits si le transfert se limite, dans un premier temps, aux ouvrages en prise avec la société et l'économie. Il propose une approche à trois niveaux: la traduction des textes fondamentaux, la vulgarisation 
intelligente de leur contenu et la traduction des outils pédagogiques susceptibles d'introduire ces savoirs dans le système éducatif.

\subsection{Défis de contexte socioculturel et politique}

Les obstacles et les défis inhérents aux sociétés arabes et qui influencent indirectement, mais largement la réussite du transfert des connaissances en arabe sont nombreux et variés. Les obstacles qui sont traités ici sont choisis soit en fonction de leur caractère inattendu, soit par le fait qu'ils n'ont pas ou peu été soulignés par les chercheurs. La reprise des défis mentionnés par ailleurs se justifie par l'urgence d'y remédier.

On ne saurait passer sous silence deux facteurs particulièrement importants: la stabilité politique et la paix sociale. Ces derniers constitueraient la condition sine qua non pour amorcer un véritable développement scientifique, dont la traduction des connaissances en arabe demeure la principale voie. Cela permettrait de réinvestir dans l'éducation au moins une partie des ressources financières et matérielles consacrées à la sécurité et à l'armement, ainsi que donner les moyens d'accomplir ce que Janjar appelle «la reprise et la consolidation du processus de modernisation culturelle qui a démarré, pour certains pays avec le mouvement de la Nahda (renaissance culturelle arabe) vers la fin du XIX ${ }^{\mathrm{e}}$ siècle» (Janjar 2013: s.p.).

Une question se pose: serait-il possible d'entamer et de réussir le transfert des connaissances mondiales vers l'arabe au vu des conflits internes et externes? De nos jours, ces conflits déchirent des pays et assassinent des populations. Les principaux centres culturels arabes, à savoir Bagdad, Damas, Tunis et Le Caire, sont particulièrement visés. Sans ces derniers, ce grand projet culturel ne serait pas réaliste. Les leçons de l'histoire et du présent nous donnent des exemples assez contradictoires: chronologiquement, l'expérience de la traduction arabe du Moyen-Âge démontre la nécessité de la stabilité politique et de la prospérité sociale. Le Siècle des Lumières témoigne du fait que traduire et développer des idées n'est pas incompatible avec la déchirure politique et l'ignorance sociale. Les expériences des pays émergents de nos jours, qui seraient l'exemple à suivre, démontrent que la majorité écrasante des pays ayant dépassé le stade de sous-développement avait joui d'une stabilité politique et sociale. La Turquie, la Chine, le Brésil, la Malaisie, l'Indonésie et l'Argentine n'auraient pas pu amorcer leur développement scientifique sans mettre fin aux dictatures et aux différents conflits qui déchiraient leurs sociétés.

Rendre à la langue arabe la place qui lui revient: la langue de l'enseignement supérieur des facultés scientifiques dans les universités arabes (Al-Saba'î 1993: 13; Al-Muhîdeb 2005: 78; Al-Haj 2009: s.p.). Voilà une question vitale pour consolider et accélérer les processus de traduction des connaissances mondiales dans la culture arabe. Cette langue n'est pas la langue d'enseignement des matières scientifiques dans la majorité des universités arabes, où est utilisé soit l'anglais, soit le français. L'utilisation de l'arabe se limite à l'enseignement des sciences humaines et sociales. Ayant fait ses preuves de lingua franca des savoirs, l'arabe doit être utilisé et généralisé comme langue d'enseignement, d'apprentissage et de recherche. À l'image de toute langue humaine naturelle, elle porte en elle le potentiel pouvant la placer au même niveau que les autres langues des sciences modernes. L'expérience de l'éducation supérieure syrienne pourrait convaincre les pessimistes et les sceptiques: la 
première faculté de médecine arabe de l'époque moderne a été créée à Damas après la Première Guerre mondiale. Depuis bientôt un siècle, l'arabe y est la langue de formation des professeurs-médecins et des apprentis médecins.

Le fait d'utiliser l'arabe comme langue de transmission et d'échanges dans les enseignements scientifiques contribue beaucoup à l'enrichissement linguistique et encyclopédique de l'arabe et aide à l'amélioration du niveau des diplômés. L'enseignement scientifique universitaire dispensé exclusivement en langue étrangère ne tient pas compte du fait que, d'une part, les apprenants concernés viennent des écoles arabophones et, d'autre part, que la langue étrangère est peu utilisée dans la pratique professionnelle sur le marché du travail arabe.

À titre d'illustration, l'enseignement est dispensé en anglais à la faculté des sciences de l'Université de Jordanie. Sans aucune mise à niveau, une étudiante admise, par exemple, en chimie doit suivre tous ses cours en anglais alors qu'elle avait étudié toutes les sciences à l'école uniquement en arabe. Sans posséder l'anglais scientifique avec sa phraséologie et sa terminologie, cette étudiante aura du mal à bien comprendre ses professeurs, surtout au début de son cursus, et passera beaucoup de temps à chercher à résoudre des problèmes linguistiques aux dépens de l'acquisition des connaissances et compétences scientifiques. Suite à un grand effort linguistique, cette apprenante finira plus ou moins bien son cursus en maîtrisant l'anglais de sa spécialité. Une fois qu'elle sera nommée professeure de sciences dans une école arabophone, on lui demandera, pour boucler la boucle, d'enseigner en arabe ce qu'elle avait appris en anglais.

L’utilisation de l'arabe dans l'enseignement supérieur «ne doit pas compromettre le niveau scientifique de l'enseignement et de la recherche universitaires, ni s'opposer à l'ouverture sur la communauté scientifique internationale» (Rahal, cité par Mahiou 2012 : 314). L'arabisation doit également s'accompagner d'un renforcement de l'enseignement des langues étrangères, notamment l'anglais et le français. Néanmoins, il ne faut pas oublier l'enseignement des langues des pays émergents, notamment le chinois et l'hindi, d'autant plus qu'il est actuellement secondaire. Par conséquent, il faudrait consolider la place de ces idiomes pour la formation des traducteurs dans ces langues et pouvoir traduire directement de ces langues, dont les pays jouent des rôles économiques et technologiques de plus en plus importants. Le dernier rapport du PNUD estime que les produits intérieurs bruts de la Chine et de l'Inde, joints à celui du Brésil, seront supérieurs à la production globale de l'Allemagne, du Canada, de la France, des États-Unis, de l'Italie et du Royaume-Uni, et constitueront en 2015, $40 \%$ du PIB mondial (PNUD 2013: iv).

L'un des chemins à emprunter pour consolider le projet de traduction des connaissances en arabe passe par l'amélioration de l'infrastructure numérique de l'arabe et l'enrichissement de son statut sur le Web. Dans une étude alarmante, Abdulrab (2009: s. p.), chercheur au CNRS en informatique, affirme que la situation de l'arabe sur le Web est déplorable. L'arabe souffre, selon les termes de ce chercheur, "de six grandes catastrophes» (Abdulrab 2009: s.p.). Trois d'entre elles concernent directement la traduction. D’abord, Abdulrab assure que l'arabe ne possède pas de logiciel de reconnaissance optique de caractères performant qui traduit les images de textes en fichiers de texte. Ce logiciel permettrait d'informatiser et de mettre en ligne toutes sortes d'ouvrages imprimés (Abdulrab 2009: s.p.). Ensuite, cet idiome manque de bases de données renfermant toutes les informations qui relèvent des 
domaines techniques et qui permettent de stocker, de retrouver et de renvoyer, via les hypertextes, à d'autres bases de données. L'absence de correcteurs d'orthographe et de moteurs de recherche adéquats est une autre faiblesse de l'informatisation de l'arabe. Ces défauts qui entravent directement la recherche terminologique et documentaire du traducteur se reflètent négativement sur les outils d'aide à la traduction, notamment les logiciels de traduction assistée par ordinateur, les mémoires de traduction et la traduction automatique.

Enfin, citons un facteur inattendu: il est communément admis que la mondialisation a entraîné la libéralisation des marchés et a contribué à une meilleure diffusion de l'information et des connaissances. Néanmoins, Jacquemond (1993) confronte les statistiques de la traduction à l'époque du président Nasser en Égypte (1955-1970), caractérisée par la fermeture économique et politique face à l'Occident, et celles de l'Infitah ${ }^{1}$, l'ouverture économique, initiée par son successeur Sadate et traduite aujourd'hui par la mondialisation. Il remarque que l'isolement politique peut être bénéfique pour la traduction vers la langue nationale.

Ce boom de la traduction [à l'époque de Nasser] s'explique d'abord, bien sûr, par le recul global de l'influence étrangère: le livre étranger devenant, matériellement et symboliquement, moins accessible, la traduction s'impose davantage comme point de passage obligé pour l'accès à la production intellectuelle étrangère. (Jacquemond 1993: s.p.)

En effet, un Arabe bilingue a tendance à lire une œuvre dans sa langue d'origine française ou anglaise et le cas échéant lire la version anglaise ou française d'un ouvrage écrit dans une autre langue plutôt que de lire sa traduction en arabe.

\section{Conclusion}

Les vifs débats et les études mentionnées montrent bien qu'il existe une prise de conscience de l'importance de la traduction, laquelle a fait évoluer la traduction d'ouvrages vers l'arabe. Les problèmes inhérents à la chaîne de traduction déjà mentionnés pourraient être résolus. Il s'agit d'investir de l'argent dans l'industrie de la traduction, et les moyens financiers ne manquent pas dans le monde arabe.

Néanmoins, la traduction des connaissances scientifiques visant l'arabophonie aurait une longue route à parcourir avant de satisfaire ses ambitions. Dans l'hypothèse où le transfert des connaissances humaines serait une entreprise réussie, le développement des sociétés arabes ne pourrait pas être envisageable sans un réel développement humain. Sans ce dernier, toute l'œuvre qui serait traduite aurait comme seule fonction d'embellir et remplir les rayons des bibliothèques arabes. Investir dans l'homme arabe passe absolument par l'instauration d'une paix sociale et d'une stabilité politique.

\section{NOTES}

1. Pour se développer, l'Égypte de Nasser avait choisi, dès la Révolution de 1952 qui avait mis fin à la monarchie, la voie de l'économie socialiste dirigée. Le président Sadate, arrivé au pouvoir en 1970, a décidé en 1973 de changer de voie et opté pour l'économie capitaliste. Il a ouvert l'économie du pays sur l'extérieur et a donné aux capitaux étrangers non seulement un accès libre à tous les secteurs économiques égyptiens, mais aussi des privilèges comme des exonérations douanières et fiscales. 


\section{RÉFÉRENCES}

Abdulrab, Habib (2009): Al-t’alîm bilgati al-'arabîa fî a-zaman alraqamî [L’enseignement de l'arabe à l'époque numérique]. Revue Al-Kalimah [Revue «Le mot»]. 29 :n.p. Consulté le 28 octobre $2010,<$ http://www.ar.aladabia.net/article-1501->.

Alecso (1997): Plan arabe pour la traduction. Organisation arabe pour l'éducation, la culture et les sciences. Tunis.

AL-HaJ, Hamîde (2009) : T'arîb at-ta'lîm al-jamî̀' [L'arabisation de l'enseignement universitaire]. L'Académie jordanienne de langue arabe (en arabe). Consulté le 6 avril 2015, <http://www. majma.org.jo/majma/index.php/2009-02-10-09-35-28/376-27-10.html>.

Al-Munîdeb, 'Abdulah (2005): T'arîb at-ta'lim al-handasî fî al-mamlâkah al-'arabîh alsa'wdîyah: al-wak' wa al-âmâl [L'arabisation de l'enseignement du génie en Arabie saoudite, réalité et perspectives]. Beyrouth: Centre d'études de l'unité arabe.

AL-SABA'î, Zuhaîr (1993): Tajrîbatî fï t'alìm attib fî al-'arabîa [Mon expérience dans l'enseignement de la médecine en arabe]. Ad-Dammām: Nâdî Al-manțiqah A-šarqîyah Al-adabî [Club littéraire de la Région de l'Est].

Baccouche, Taïeb (2000) : La traduction dans la tradition arabe. Meta. 45(3):395-399.

BADRAN, Adnan (2006). Rapport de l'UNESCO sur la science: les États arabes. New York: Éditions de l'UNESCO. Consulté le 3 mai 2013, <http://www.unesco.org/new/fileadmin/ MULTIMEDIA/HQ/SC/pdf/sc_usr05_arab_fr.pdf $>$.

Basalamah, Salah (2011): Les défis traductifs de l'islam post-11/9. Global Media Journal. 4(2):115-124. Consulté le 18 mars 2015, <http://www.gmj.uottawa.ca/1102/v4i2_basalamah. $\mathrm{pdf}>$.

Beligh, Nabli (2013): Comprendre le monde arabe. Paris: Armand Colin.

Cheddadi, Abdesselam (2009): Traduction et culture dans le monde arabe. Revue Esprit. 12. Consulté le 3 octobre 2013, <http://www.esprit.presse.fr/archive/review/article.php?code $=14767>$.

Delisle, Jean et Woodsworth, Judith, dir. (1995): Les traducteurs dans l'histoire. Ottawa: Presses de l'Université d'Ottawa.

Dessa, Hasnaa et JANJAR, Mohamed-Sghir (2010): État de la traduction arabe des ouvrages de sciences humaines et sociales 2000-2009. In: Ghislaine Glasson Deschaumes, dir. État des lieux de la traduction en Méditerranée. Paris/Casablanca: Transeuropéennes/Fondation du Roi Abdul Aziz. Consulté le $1^{\text {er }}$ mai 2013, <http://www.transeuropeennes.eu/ressources/ pdfs/TEM2010_SHS_monde_arabe_Hasnaa_DESSA_Mohamed_Sghir_JANJAR_38.pdf>.

Glasson Deschaumes, Ghislaine (2012): L'état des lieux de la traduction en méditerranée. Transeuropéennes et Fondation euro-méditerranéenne Anna Lindh. Consulté le 17 mars 2015, <http://www.transeuropeennes.eu/ressources/pdfs/TEM_2012_Conclusions_et_ recommandations_116.pdf $>$.

JaCquemond, Richard (1993): Traductions croisées Égypte-France: stratégies de traduction et échange culturel inégal. Revue Égypte/Monde arabe. 15: s.p. Consulté le 18 octobre 2013, $<$ http://www.ema.revues.org/index1109.html>.

JACQUEMOND, Richard (2007): Les Arabes et la traduction: petite histoire d'une idée reçue. La pensée de midi. 21:177-184.

JACQUEMOND, Richard (2012): État des lieux de la traduction dans la région euro-méditerranéenne: traduire de et vers l'arabe, synthèse. Transeuropéennes. Consulté le 13 mars 2015, <http:// www.transeuropeennes.eu/ressources/pdfs/TEM_2012_Synthese_sur_la_traduction_de_ et_vers_l_arabe_123.pdf $>$.

JANJAR, Mohamed-Sghir (2013) : Traduction et constitution de nouveaux champs des savoirs en langue arabe. Transeuropéennes. Consulté le 20 avril 2013, <http://www.transeuropeennes. eu/fr/articles/voir_pdf/221>.

Mahiou, Ahmed (2012): Réforme de l'enseignement supérieur en Algérie. In: Jean-Robert Henry et Jean-Claude Vatin, dir. Le temps de la coopération: sciences sociales et décolonisation au Maghreb. Paris: Karthala, 314-320. 
Ministère des Affaires Étrangères (MAE) franÇAis (2013). Consulté le 23 avril 2013, $<$ http://www.tradarabe.org/index.php?p=24>.

Naggar, Mona (2011): Traduction de l'allemand vers l'arabe. Transeuropéennes. Consulté le 25 octobre 2013, <http://www.transeuropeennes.eu/ressources/pdfs/TIM_2011_German _Arabic_Mona_NAGGAR_French_version_109.pdf >.

ODEH, Akram (1998): La traduction et la terminologie linguistique du français en arabe: l'arabisation du Cours de linguistique générale de F. de Saussure. Thèse de doctorat non publiée. Lyon: Université de Lyon II.

PNUD (1999): Rapport mondial sur le développement humain 1999. New York: Publications des Nations Unies. Consulté le 27 mars 2013, <http://www.arab-hdr.org/publications/other/ undp/hdr/1999/hdr-f.pdf $>$.

PNUD (2003): Rapport sur le développement humain dans le Monde arabe. New York: Publications des Nations Unies. Consulté le 27 mars 2014, <http://www.arab-hdr.org/publications/ other/ahdr/ahdr2003e.pdf >.

PNUD (2013): Rapport mondial sur le développement humain. New York: Publications des Nations Unies. Consulté le 28 octobre 2013, <http://www.undp.org/content/dam/undp/ library/corporate/HDR/2013GlobalHDR/French/HDR2013\%20Report\%20French.pdf>.

Salama-CARr, Myriam (1990): La traduction à l'époque abbasside. Collection Traductologie. Vol. 6. Paris: Didier Érudition, $122 \mathrm{p}$.

SHAWQI, Galal (1999): Al-Tarjama fîl-'âlam al-'arabî, al-wâqi' wa-l-tahaddî [La traduction dans le Monde arabe: l'état des lieux et les défis]. Le Caire: Conseil supérieur de la culture (en arabe).

YAsA, Alparslan (2003): La traduction chez les Turcs Anciens et sous la dynastie Abbasside. Hacettepe Üniversitesi Edebiyat Fakültesi Dergisin. 3:203-223.

ZAKARIYA, Fouad (1990): Les racines culturelles du sous-développement intellectuel arabe. Revue Égypte/Monde arabe. 3: s.p. 